\title{
Optimal management of ankle syndesmosis injuries
}

This article was published in the following Dove Press journal:

Open Access Journal of Sports Medicine

5 August 2014

Number of times this article has been viewed

\section{David A Porter \\ Ryan R Jaggers \\ Adam Fitzgerald Barnes \\ Angela M Rund}

Methodist Sports Medicine/The Orthopedic Specialists, Indianapolis, IN, USA

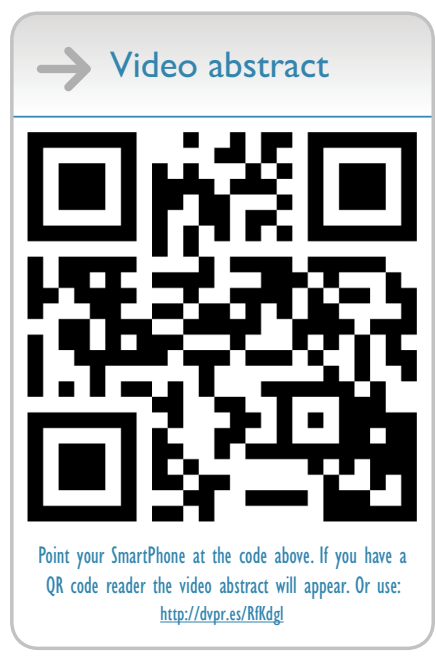

Correspondence: David A Porter Methodist Sports Medicine/The Orthopedic Specialists, 20I Pennsylvania Parkway, Suite 100, Indianapolis, IN 46280, USA

Tel + I 317817 I285

Email dporter@methodistsports.com
Abstract: Syndesmosis injuries occur when there is a disruption of the distal attachment of the tibia and fibula. These injuries occur commonly (up to $18 \%$ of ankle sprains), and the incidence increases in the setting of athletic activity. Recognition of these injuries is key to preventing long-term morbidity. Diagnosis and treatment of these injuries requires a thorough understanding of the normal anatomy and the role it plays in the stability of the ankle. A complete history and physical examination is of paramount importance. Patients usually experience an external rotation mechanism of injury. Key physical exam features include detailed documentation about areas of focal tenderness (syndesmosis and deltoid) and provocative maneuvers such as the external rotation stress test. Imaging workup in all cases should consist of radiographs with the physiologic stress of weight bearing. If these images are inconclusive, then further imaging with external rotation stress testing or magnetic resonance imaging are warranted. Nonoperative treatment is appropriate for stable injuries. Unstable injuries should be treated operatively. This consists of stabilizing the syndesmosis with either trans-syndesmotic screw or tightrope fixation. In the setting of a concomitant Weber B or C fracture, the fibula is anatomically reduced and stabilized with a standard plate and screw construct. Proximal fibular fractures, as seen in the Maisonneuve fracture pattern, are not repaired operatively. Recent interest is moving toward repair of the deltoid ligament, which may provide increased stability, especially in rehabilitation protocols that involve early weight bearing. Rehabilitation is focused on allowing patients to return to their pre-injury activities as quickly and safely as possible. Protocols initially focus on controlling swelling and recovery from surgery. The protocols then progress to restoration of motion, early protected weight bearing, restoration of strength, and eventually a functional progression back to desired activities.

Keywords: syndesmosis, athletes, deltoid ligament, operative fixation, rehabilitation

\section{Introduction}

Syndesmosis injuries involve the distal tibiofibular joint and can disrupt the normal stability of the ankle joint depending on their severity. This instability, if uncorrected, can lead to chronic instability and significant morbidity, ultimately leading to degenerative arthritis. The ultimate goal with syndesmotic injury is a return to full activities as rapidly and safely as possible. This may involve operative or nonoperative management, followed by a structured rehabilitation program. After initial treatment, the patient can expect a 2-6-month recovery period before return to pre-injury activities. The keys to treating syndesmosis injuries are 1) appropriate recognition of the injury and its severity, 2) assessment of the degree of instability, 3) reduction and stabilization of 
the ankle joint, and 4) progressive rehabilitation focusing on full range of motion and normal strength.

\section{Epidemiology}

Most recent sources indicate that syndesmosis injuries constitute $1 \%-18 \%$ of all ankle sprains. ${ }^{1-3}$ Within the athletic population, the incidence of injury increases from $12 \%$ to $32 \% .{ }^{4,5}$ Of these athletes, most participate in sports that involve planting the foot and cutting, or blows to the outside of the ankle forcing external rotation and concomitant plantarflexion or dorsiflexion. ${ }^{6}$ Additionally, athletes tend to display aggressive behavior, shorter resting periods, and greater risktaking behavior, all of which increase the risk of sustaining such an injury. ${ }^{4}$ With various sports, the frequency of ankle injuries that involve the syndesmosis increases, peaking in professional hockey at $70 \% .^{7}$ Despite the prevalence of unstable injuries in both the general and athletic population, these injuries are often misdiagnosed as a stable high ankle sprain. This misdiagnosis of severity can lead to increased morbidity, slower rehabilitation, and arthritic changes later in life. ${ }^{8}$ Generally, syndesmosis injuries also take twice as long for recovery as compared with lateral ankle sprains of a similar grade. ${ }^{2}$ With so many factors that impede a successful recovery, optimal management is essential.

\section{Anatomy and physiology}

The syndesmosis provides stability for the articulation of the tibia and fibula. The distal fibula rests between the anterior and posterior lateral tibial tubercles to provide bone stability and normally only widens $1 \mathrm{~mm} .{ }^{9}$ Furthermore, if the Chaput's tubercle is too shallow, the patient may be predisposed to recurrent external rotation ankle sprains. ${ }^{10}$ At the distal end of the fibula, these two bones are connected by four primary ligaments: the anterior-inferior tibiofibular ligament (AITFL), the posterior-inferior tibiofibular ligament (PITFL), the interosseous ligament (IOL), and the deltoid ligament. ${ }^{11}$ In syndesmosis injuries, the AITFL and the anterior deltoid ligaments are the first to tear. ${ }^{3}$ The IOL connects the tibia and fibula proximally through their lengths and helps provide stability and support to the ankle joint. The IOL acts as a spring to allow slight separation during dorsiflexion. ${ }^{12}$ The PITFL is the last to tear due to its combination of strength and elasticity and that the deforming force is external rotation. Cadaveric sectioning has revealed the stability contribution of each ligament to be $35.5 \%$ AITFL, 32.7\% deep PITFL, $8.7 \%$ superficial PITFL, and $21.6 \%$ IOL. ${ }^{12}$ Sectioning of the AITFL causes the greatest amount of diastasis in the joint. Tears to all three ligaments results in $7.3 \mathrm{~mm}$ of widening and an increase in external rotation by $10.2^{\circ} .{ }^{13}$ Syndesmosis injuries involve a tear to one or more of these ligaments, in addition to at least a portion of the deltoid ligament. ${ }^{12}$

The deltoid ligament is twice as strong as the lateral ligaments and provides stability to the ankle mortise. ${ }^{2}$ The deep deltoid prevents eversion and lateral displacement of the talus, while the superficial deltoid limits external rotation. ${ }^{14}$ While no unanimous approach has been adopted concerning the repair or nonoperative treatment of the deltoid ligament, its importance for ankle stability cannot be denied. The articulation between the fibula and talus prevents talar shifting, of which a $1 \mathrm{~mm}$ shift has been shown to decrease tibiotalar contact area by $42 \%$ and increase weight loads. ${ }^{15}$ Additionally, a lateral talar displacement greater than $2 \mathrm{~mm}$ may result in greater than $90 \%$ chance of degenerative joint changes if left unaddressed because of increased pressure with decreased contact area. ${ }^{16}$

\section{History}

Since syndesmosis injuries often present with confounding symptoms similar to severe lateral ankle sprains, an understanding of the mechanism of injury is crucial. Furthermore, the ability to distinguish between the two injuries is essential in order to provide optimal management; a grade III lateral ankle sprain (treated nonoperatively) can appear similar to an unstable syndesmosis injury (treated operatively). Knowing the mechanism of injury is the first step toward differentiating between the two. Location of injury, type of sport, position of limb, and ability to bear weight can all provide additional clues toward a proper diagnosis. Noting the elapsed time since the incident and the corresponding amounts of pain and swelling also aid in recognition of a syndesmosis injury. Compared with patients who have sustained a grade III lateral ankle sprain, patients with a syndesmotic injury often report instability and difficulty ambulating. Understanding pre-injury levels of activity also helps to set expectations for recovery and create an appropriate management plan. On the initial visit, a general medical screen is required, consisting of questions concerning diabetes, vascular disease, neuropathy, alcohol use, and medication use. This screening process can uncover conditions that affect the timing of surgical intervention and the rehabilitation course, such as length of immobilization, concerns for soft tissues, risk for infection, and likely compliance. ${ }^{8}$

\section{Classification}

Syndesmosis injuries involve the disruption of the connecting tibiofibular ligaments in addition to the disruption of the 
deltoid ligament medially. ${ }^{8}$ The most common disruption results from forceful internal rotation of the leg and external rotation of the talus with a planted foot. ${ }^{7}$ There can be a concomitant plantarflexion or dorsiflexion at the ankle. This external rotation movement widens the ankle mortise and tears the AITFL, which allows the fibula to separate from the tibia. The lateral talar movement and eversion can also injure the medial deltoid ligament. Depending on the severity of the injury, a proximal fibular fracture may also be present (Maisonneuve fracture).

The two most utilized classification systems for ankle sprains and fractures are the graded ankle sprain and the Danis-Weber classification system of fibula fracture. ${ }^{8}$ The graded system classifies ankle instability according to mechanism of injury, ankle instability, and degree of ligament disruption. Grade I injuries involve partial tears of the AITFL, the anterior deltoid ligament, and distal IOL. Since there is no diastasis present, the ankle is considered stable. ${ }^{8}$ In grade II injuries, the rotational force tears the anterior and deep deltoid ligaments and the AITFL, and partially tears the IOL. ${ }^{3}$ Grade II sprains can be difficult to diagnose on radiographs and are often classified as latently unstable syndesmosis injuries. Without stress radiographs, the tibiofibular diastasis is not identified. Underestimating the severity of injury can lead to further injury, interosseous ossification, chronic pain and stiffness, and other sequelae. ${ }^{17}$ If not diagnosed promptly, an acute syndesmosis injury can turn into a chronic problem. Chronic syndesmosis injuries, defined as no treatment greater than 3 months after the injury was sustained, leads to inevitable arthritis, higher rates of ligament ossification, and poorer functional outcomes. ${ }^{1,18,19}$ Grade III injuries involve complete disruption of the syndesmosis and frank instability. ${ }^{1}$

Forceful external rotation involved in this classification of injury can result in concomitant fibular fractures. The three most common associated fractures include Weber B, Weber C, and Maisonneuve fractures. Weber B fractures occur at the level of the distal syndesmosis (ankle joint), and are caused by an external rotation mechanism. These often do not have a disruption of the interosseous membrane. Weber $\mathrm{C}$ fractures involve disruption of the deltoid ligament caused by external rotation and a fibular fracture above the level of the distal syndesmosis (ankle joint). ${ }^{16}$ Weening and Bhandari ${ }^{20}$ reported that $70 \%$ of patients suffering from a syndesmosis injury with an associated fracture experience a type $\mathrm{C}$ fracture. Maisonneuve fractures are characterized by a proximal fibula fracture. The more proximal the fibular fracture, the greater the risk of displaying a syndesmosis disruption and associated instability.

\section{Clinical examination}

Upon presentation of a possible syndesmosis injury, if the patient walks with decreased power at push-off, complains of localized pain of the AITFL that is tender to the touch, and/or experiences pain with external rotation and dorsiflexion, the physician should suspect a syndesmosis injury. ${ }^{2}$ Soft tissue damage, ecchymosis, and tenderness to palpation over the medial or lateral malleolus can direct the physician in determining the extent of injury also. The entire fibula must be palpated to rule out a proximal Maisonneuve fracture. ${ }^{8}$ Fites et $a{ }^{17}$ also advised suspicion for a syndesmosis injury when swelling above the joint line between the tibia and fibula occurs within the first 24 hours after sustaining the injury. Sometimes tenderness of ligamentous injuries can be isolated to specific regions of the ankle. Deltoid damage can be assessed by testing the presence of pain over the medial malleolus and just distal to it. ${ }^{21}$ Difficulties in differentiating between the deep deltoid ligament and posterior syndesmotic ligaments can complicate the diagnosis of severity. Nussbaum et $\mathrm{al}^{19}$ reported that the distance of interosseous tenderness and a positive squeeze test correlates well with days lost from competition and the severity of ligamentous damage. In situations like these, repeat evaluations or radiography may be necessary. Rapid and accurate identification of the injury can reduce the risk of poor outcomes and life-long morbidity associated with chronic syndesmosis injuries.

The most notable physical examination tests used to assess the extent of injury include the external rotation test and squeeze test. To perform an external rotation test, the patient is seated with the hips and knees flexed to $90^{\circ}$. One of the examiner's hands is used to stabilize the lower leg, while the other hand is used to externally rotate the foot. If pain is observed, a syndesmosis injury is suspected. This movement replicates the mechanism of injury and has been documented as the most reliable test and displays low interobserver error and high sensitivity. ${ }^{22}$ The squeeze test may also provide a useful diagnostic tool. ${ }^{9}$ In this test, the mid-calf is squeezed over the tibia and fibula to see if pain is elicited distally at the ankle. The authors warn that they have not found the squeeze test to be as reliable when evaluating a syndesmosis injury.

During physical examination, the physician should check the amount of rotation permitted at the ankle joint as well as the amount of diastasis as assessed by radiographic imaging. The amount of diastasis and rotation has been shown to be related to the amount of ligamentous damage. ${ }^{13}$ 


\section{Radiography}

When radiographically examining the ankle joint, weightbearing anterior-posterior, lateral, and mortise views of the ankle joint should be obtained. If necessary, injections of local anesthetic may be used around the ankle joint to minimize discomfort and allow the stress of full weight bearing. ${ }^{17}$ When required, $1 \%$ lidocaine was used in the deltoid $(3-5 \mathrm{~mL})$ and distal syndesmosis $(4-6 \mathrm{~mL})$. Xenos et al ${ }^{13}$ determined stress lateral radiographs to be more accurate at assessing diastasis than stress mortise radiographs. However, Lin et $\mathrm{al}^{2}$ found weight-bearing mortise radiographs to be the best assessment of ankle instability. Since no agreement exists in the literature, all three views should be examined carefully. In all views, the authors prefer physiological stress to help evaluate occult deltoid and syndesmotic ligament injuries. This helps to differentiate between an isolated lateral malleolus fracture and a bimalleolar equivalent injury and between grade I and grade II injuries. Stress external rotation views are obtained if the weight-bearing films are inconclusive regarding stability (Figure 1).

Indications of a syndesmosis injury are assessed via the amount of medial clear space, widening of the tibiofibular clear space, and tibiofibular overlap. ${ }^{23} \mathrm{~A}$ syndesmosis injury is present when the ankle joint exhibits greater than $1 \mathrm{~mm}$ lateral subluxation or greater than $5 \mathrm{~mm}$ separation between the distal fibula and tibia on the mortise view. ${ }^{24}$ Poor results are associated with a difference in syndesmotic width greater than $1.5 \mathrm{~mm}$ compared with the contralateral side. ${ }^{25}$ Medial clear space is the most accurate evaluation of diastasis, but physicians should be hesitant to make conclusions solely based off radiographic images. Ankle diastasis is difficult to determine via X-ray imaging due to the variability of tubercle sizes, fibular notch depths, and positioning. ${ }^{1}$

If plain radiographs do not provide adequate evidence of a suspected syndesmosis injury, further evaluation with ultrasound, computed tomography (CT) scans, or magnetic resonance imaging (MRI) should be employed. Ultrasound offers a quick and inexpensive form of assessment of the ankle without the hazards of other imaging devices. However, it is user dependent and lacks the fine details provided by CT or MRI. ${ }^{1} \mathrm{CT}$ scans are excellent for evaluating fine bony details. MRI has the ability to provide fine anatomic detail, including information about soft tissue structures. Crim et $\mathrm{a}^{14}$ documented the utility of MRI in detecting deltoid and osteochondral injuries. In their study, MRI scans detected 15/16 osteochondral lesions without any false positives when compared with control of arthroscopic evaluation. MRI is also the preferred imaging modality in the setting of

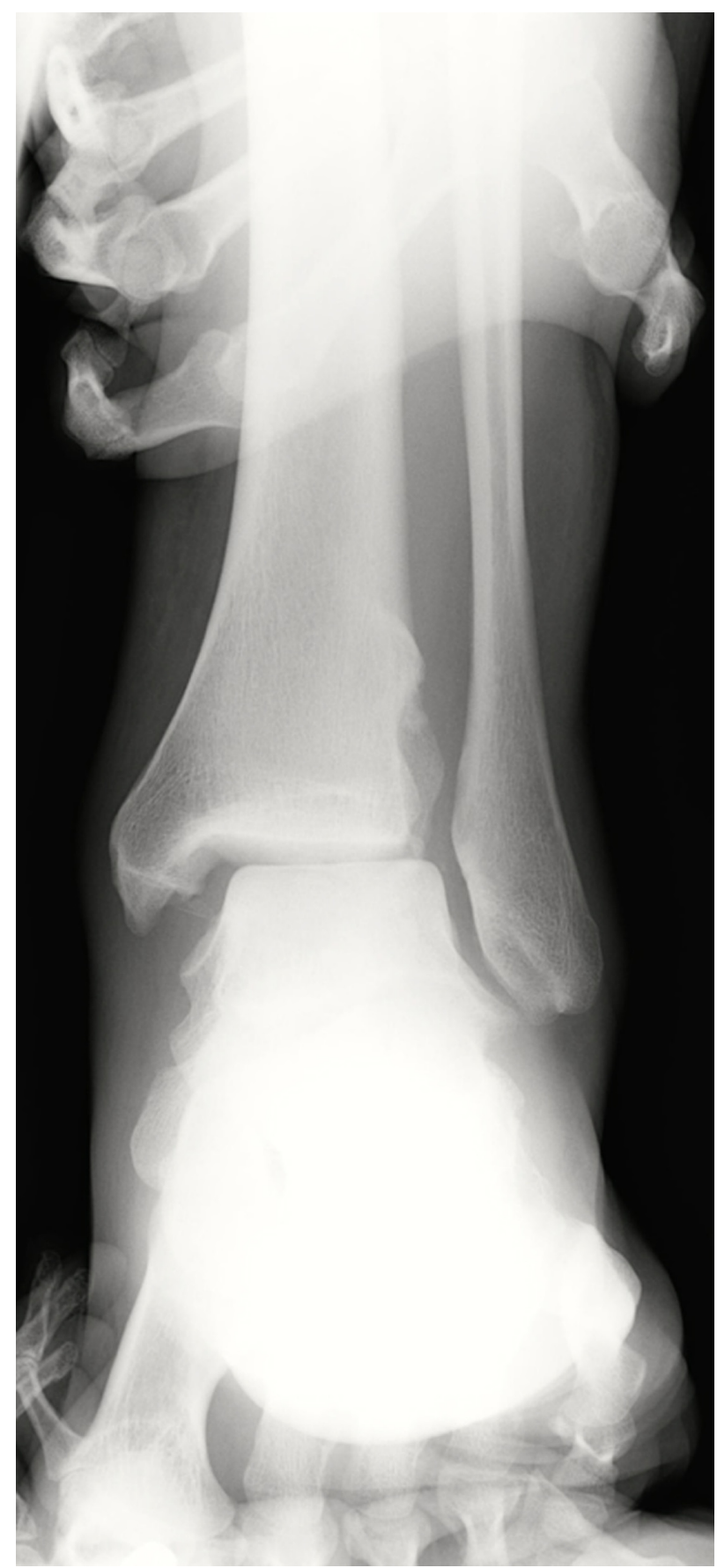

Figure I Abduction and external rotation anterior-posterior stress image of left ankle demonstrating unstable syndesmosis and wide medial clear space.

chronic injuries, as it provides very high sensitivity (90.0\%) and specificity $(94.8 \%) .{ }^{26}$

Imaging modalities also play a crucial role during the operative treatment of these injuries. Intraoperative fluoroscopy has long been used for immediate confirmation of reduction and fixation placement. However, a recent study by Franke et $\mathrm{al}^{27}$ using intraoperative three-dimensional imaging questions the accuracy of fluoroscopy and advocates for 
more advanced intraoperative imaging. In their study, $32.7 \%$ of fractures that appeared appropriately reduced on fluoroscopy were found to be malreduced, most commonly anterior displacement and internal rotation of the distal fibula.

\section{Management plan}

Objectives to follow during a treatment plan include providing anatomic reduction of any deformities or fractures, care of soft-tissue damage, repair of associated injuries, rehabilitation, and treatment of any complications that may arise. Of the various classes of injury, grade I treatments are the only degree of injury that should be treated nonsurgically. Upon diagnosis, boot immobilization and cryotherapy are employed. ${ }^{8}$ On subsequent visits, radiographs should be obtained to ensure proper alignment until stability is confirmed. Generally, patients use crutches for 1 or 2 weeks and then progressively begin weight bearing during the next 4 weeks. In the instances of deltoid damage, early stress may stretch or disrupt proper healing of the ligament, so this must be kept in mind. ${ }^{9}$ Thus, after the first 2 weeks, the patient is weaned into an off-the-shelf stirrup brace or a custom short articulating ankle-foot orthosis with correction for any pronation. Gradually, the patient commences range-ofmotion and stretching exercises followed by a bike program and balance exercises. The average time to return to sports ranges between 4 and 8 weeks. ${ }^{8}$

For all grade II and III injuries, these authors recommend surgical intervention. All grade II and III injuries are considered unstable and must be repaired to regain previous functional ability. ${ }^{28}$ Weening and Bhandari ${ }^{20}$ identified adequate reduction as the sole significant indicator of both short musculoskeletal function assessment outcome scores and the ability to run after recovery. At the time of surgery, the physician should remove any loose fragments or debris within the articular space. ${ }^{8}$

\section{Operative treatment}

In all cases of grade II or III injuries, surgical reduction and internal fixation is the recommended treatment, especially in the setting of young and/or athletic patients. All surgical treatments of syndesmotic injuries rest on the key principle of anatomic reduction of the ankle joint and secure, anatomic fixation of the distal tibiofibular joint. Reestablishing the correct tibia-fibula interval, fibula length, and proper alignment of the fibula in the tibial incisura are critical for a good outcome. The syndesmosis is held in place with the use of a large reduction clamp compressing the tibia and fibula. When placing this clamp, care must be taken to place it in a neutral anatomic axis to avoid oblique malreduction. ${ }^{29}$ Due to the talus being wider anteriorly than posteriorly, it has long been recommended that fixation be performed with the foot in dorsiflexion to avoid over-constraining the joint. However, studies have shown that the position of the foot does not affect postoperative range of motion. ${ }^{30,31}$ The present authors advise placing screw fixation with the foot in neutral dorsiflexion, but allowing mild plantarflexion of the ankle when using suture button fixation to allow anatomic reduction and the ability to allow for creep with suture buttons.

A point of contention of these injuries is the necessity of reconstructing/repair of the deltoid ligament. Some physicians maintain the opinion that if adequate reduction of the syndesmosis joint and talar positioning is obtained from an anterolateral approach and cast immobilization is used to place the foot in slight plantarflexion and inversion, deltoid reconstruction is unnecessary. ${ }^{3,21}$ If reduction cannot be obtained, it necessitates deltoid exploration on the medial side. Strömsöe et al ${ }^{32}$ found that in 50 patients with either concomitant Weber B or Weber C fractures, deltoid ligament repair did not improve functional outcome, fibular reduction, or a quicker return to weight-bearing. However, the present authors implement an aggressive rehabilitation program in which weight-bearing commences approximately 2 weeks after surgery. The stress of earlier weight-bearing on a non-repaired deltoid is unknown. The present authors also prefer to perform a deltoid repair for three additional reasons: 1) the joint can be evaluated from the medial side to visualize potential articular chondral or osteochondral lesions; 2) deltoid repair mitigates joint laxity and potentially hastens the ability to bear weight; and 3) the deltoid contributes largely toward sustaining medial stability, thus primary repair gives more assurance to maintaining this anatomic length to the deltoid. We realize that our recommendation in this article is based on our own experience, but we have used this approach for over 15 years and find it very useful. Others will have a different opinion on this deltoid treatment.

A "Brostrom-like" procedure with two \#1 Vicryl horizontal mattress sutures in the deep deltoid and two \#0 Vicryl sutures in the superficial (anterior) deltoid are used to repair the deep and then superficial deltoid ligaments. We place our sutures in the deep deltoid before we go laterally to fix the fibular or syndesmosis since it is much easier to access when the whole joint is unstable. If there is insufficient tissue for a "Brostrom-like" repair, an autologous graft can be taken from the plantaris, the second or third toe extensor, the semitendinosus, or the gracilis. If needed, these autografts can be used to repair the syndesmosis ligaments as well. The present authors 
prefer an autogenous gracilis graft in the chronic injury only. Since the deltoid ligament is essential for the stability of the ankle mortise, deltoid ligament repair helps to mitigate against future laxity. ${ }^{1,2,8,9}$ Hintermann et $\mathrm{al}^{28}$ advised surgical reconstruction of the deltoid to restore mechanical stability. After an average follow-up of 4.4 years, they found American Orthopaedic Foot and Ankle Society (AOFAS) scores equal to 91.6 on various degrees of deltoid tears; original AOFAS scores equaled 42.9. Furthermore Crim et $\mathrm{al}^{14}$ determined a deltoid tear to be associated with chronic ankle instability.

If osteochondral lesions are present while examining the deltoid ligament integrity, they should be debrided and microfractured. However, care should be taken to operate minimally on the bone, as too much excision can disrupt the weight-bearing surface and lead to unfavorable outcomes. ${ }^{8}$

\section{Screw fixation}

Fixation with trans-syndesmotic screws has long served as a reliable method of tibiofibular stabilization. A recent study by Lambers et al $^{33}$ examined 21-year follow-up in patients who underwent treatment with two syndesmotic screws for Maisonneuve-type pronation-external rotation injuries. They found that while nearly half of the patients had radiographic evidence of arthritis, 92\% had good or excellent functional outcomes. ${ }^{33}$ In cases using syndesmotic screws, no study has found statistical differences between quadricortical and tricortical screws or the number of screws used. ${ }^{25} \mathrm{~A}$ fourhole, one-third tubular plate can be positioned over the lateral fibula and secured with $3.5 \mathrm{~mm}$ screws in the most proximal and distal holes stabilizing the placement. Under fluoroscopic guidance, a partially threaded $4.5 \mathrm{~mm}$ screw is placed across the syndesmosis, $1 \mathrm{~cm}$ above the tibial plafond. A second $4.5 \mathrm{~mm}$ screw is drilled proximally (fully threaded) for additional stability. In the rare instance of a Weber B pattern with an unstable syndesmosis, the present authors use anterior-to-posterior lag screw fixation and a posterolateral one-third tubular antiglide plate. Weber type $\mathrm{C}$ fractures are stabilized by a lateral slide plate and occasionally a $2.7 \mathrm{~mm}$ or $3.5 \mathrm{~mm}$ lag screw. True bimalleolar (medial malleolus and lateral malleolus) and bimalleolar equivalent (lateral malleolus and deltoid rupture) fractures follow anatomic reduction as prescribed via Weber type B and type C procedures, with additional repair of the deltoid ligament. True bimalleolar and bimalleolar equivalent fractures are classified together because the prognosis, recovery, rehabilitation, and decision-making are similar. ${ }^{8}$ One or two plates may be used depending on the extent and location of fracture. If a Maisonneuve fracture is present, fibular fixation is not performed for the proximal fibula fracture component. The syndesmotic screws and plate are typically removed 2-4 months after surgery. Some advocate screw removal should be delayed in overweight patients to ensure adequate reduction is maintained. ${ }^{2}$ Currently, the present authors stabilize syndesmosis injuries associated

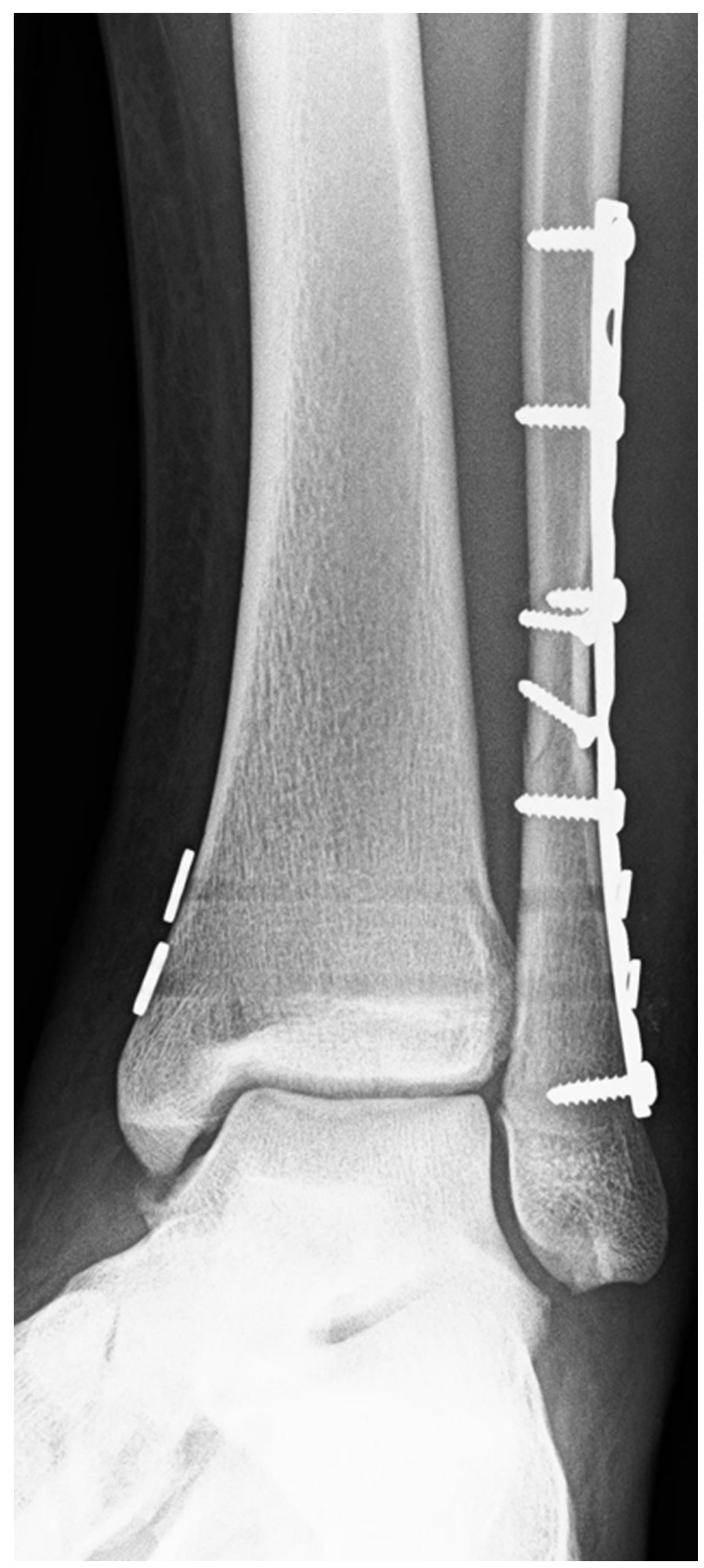

Figure 2 Postoperative left anterior-posterior ankle radiograph after open reduction and internal fixation of Weber $\mathrm{C}$ fibular shaft fracture with lag screws, one third tubular plate and two suture button fixation of the syndesmosis demonstrating anatomic alignment of the syndesmosis, medial clear space and fibular shaft. The deltoid ligament was also repaired in this athlete. 
with a Weber $\mathrm{C}$ fracture with a suture-button device through the same plate used for the fibula fracture fixation (Figure 2). With this technique, there is no planned hardware removal. We ensure medially that the endobutton is subperiosteally placed to decrease "creep" and decrease the risk of tissue necrosis under the button leading to osteomyelitis.

Previous studies utilizing screw fixation have obtained good results. Taylor et $\mathrm{al}^{24}$ reported on six collegiate athletes who had excellent subjective ratings on an average 34 months after surgery. They also noted an average return to sports 41 days after surgical treatment and rehabilitation, and that prolonged immobilization led to increased stiffness in the joint. ${ }^{24}$ Wikerøy et $\mathrm{al}^{25}$ found a significant difference in dorsiflexion between the involved and noninvolved ankles, and lower subjective ratings from obese patients or patients displaying arthritis. The literature shows that more than half of patients undergo a secondary operation to remove screws. ${ }^{20,34}$ However, patients with retained screws that are broken or loose have similar outcomes to those undergoing screw removal and better results than those with retained intact screws. ${ }^{35}$

In the setting of chronic syndesmosis injuries, arthrodesis of the syndesmosis may be advisable. Arthrodesis is a salvage operation and should only be utilized in cases of painful arthritis or chronic instability. ${ }^{3}$

\section{Suture-button fixation}

Recent years have seen the advent of suture-button devices, providing what has proven to be another viable option for syndesmosis stabilization. Suture-button fixation consists of a braided, nonabsorbable fiberwire suture that spans the distal tibiofibular articulation that is secured in place directly on the bone on each side by an endobutton (or over the plate in the setting of fibular fracture fixation). This provides for potential advantages over screw fixation, including greater, more anatomic mobility of the joint, quicker return to weight bearing and sports, no osteolysis, and no need for hardware removal. ${ }^{17,21,36,37}$ The procedure is performed with the same basic principles as screw fixation. After anatomic restoration of the syndesmosis and placement of a tibiofibular clamp, a $2.7 \mathrm{~mm}$ drill is used to create the intraosseous pathway. A guidewire is then used to pull one endobutton and the suture through to the medial side. The endobutton is toggled and flipped, and the suture is tensioned and secured laterally over a second endobutton, which is placed over a lateral fibular plate. Newer designs with knotless tightrope systems have helped to minimize the risk of soft tissue irritation over the lateral knot. ${ }^{38}$ In the setting of purely ligamentous injuries, the present authors have recently moved to a combination

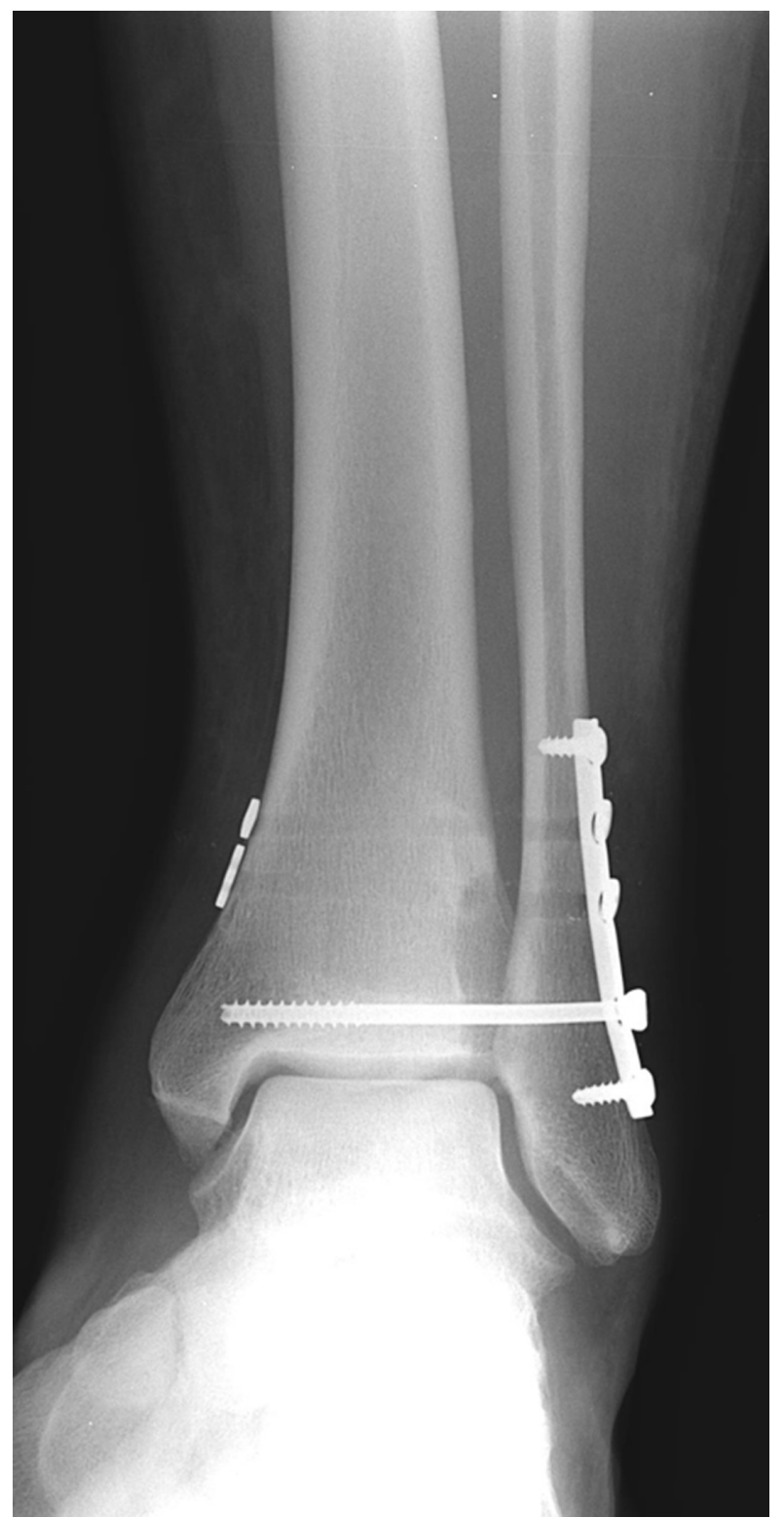

Figure 3 Postoperative anterior-posterior ankle radiograph after open reduction and internal fixation of unstable syndesmosis injury with five hole one third tubular plate, two suture buttons and one $4.5 \mathrm{~mm}$ cannulated screw demonstrating anatomic alignment of the syndesmosis and the medial clear space. The deltoid was also repaired.

of screw and suture-button fixation (Figure 3). This allows for initial rigid fixation until screw removal, which can be performed in the office. The suture-buttons then remain for longer-term stabilization.

Results of suture-button treatment have also been favorable. In Naqvi et al's ${ }^{38} 2012$ study, 42 of 49 patients reported very good to excellent results with weight-bearing by 7.7 weeks and return to normal activity at 11.2 weeks on average; these results are comparable to screw fixation. Willmott et a ${ }^{39}$ reported skin irritation and subsequent implant removal in two of six patients using the tightrope device. The 
mean time to weight-bearing was 6 weeks, and they attributed the need for removal to the fact that in those two patients they placed the tightrope directly through the fibula rather than through a plate. Cottom et $\mathrm{al}^{40}$ also described 23 of 25 patients with good-excellent subjective scores after a suture-button procedure. Return to full weight-bearing took 5.5 weeks. Qamar et $\mathrm{al}^{37}$ found no loosening of the tightrope device in 16 patients at a 2-year follow-up and full weight-bearing and return to work took 4.5 weeks and 8.4 weeks on average, respectively. This evidence provides good news for obese or elderly patients who may have concerns with increased stress on the device or arthritic changes.

\section{Screw fixation versus suture- button fixation}

There seems to have been a recent shift toward suture-button fixation by many physicians. This has largely been due to the belief that it provides quicker weight bearing, shorter rehabilitation, and better functional outcomes compared with screw fixation. ${ }^{38}$ In a comparative study between tightrope and screw fixation groups (23 patients each), Naqvi et $\mathrm{al}^{38}$ determined screw fixation to allow significant diastasis of the involved syndesmosis in comparison with the contralateral side and $21.7 \%$ greater incidence of hardware removal. No significant difference existed for return to full weight-bearing, although the tightrope group returned a week earlier on average. Thornes et $\mathrm{al}^{41}$ reported significantly better AOFAS scores for suture-button patients in comparison with screw fixation (91 versus 80; 93 versus 83) at 3-month and 1-year follow-ups, and a faster return to work (2.8 months versus 4.6 months). Furthermore, $75 \%$ of the screw fixation patients needed hardware removal, while none of the suture-button patients required a second surgery. ${ }^{41}$ Finally, in a systematic review, Schepers ${ }^{34}$ determined that patients undergoing a suture-button procedure returned to work earlier, exhibited slightly higher AOFAS scores, and required a second surgery less often ( $22 \%$ versus $52 \%$ ). It is based on these results that these authors uses a suture-button device to reduce syndesmosis injuries as well as Weber $\mathrm{C}$ fractures. If a bimalleolar fracture occurs, screw fixation may be necessary to reduce the fibula. Especially with respect to athletes, a suture-button device is preferred. A tightrope device provides greater mobility within the ankle joint and a quicker return to sport. A higher incidence of syndesmosis injuries occurs in the athletic population, with a majority of those injuries occurring in football. ${ }^{4,19}$ The elimination of a subsequent surgery, especially in this population, makes the suture-button device the present authors' preferred method of reduction.

\section{Postoperative rehabilitation}

Aggressive rehabilitation with early range of motion and weight-bearing exercises allows patients to regain functional activity at the quickest rate. Mobility and strengthtraining exercises promote the natural healing process of the syndesmosis and associated ligaments, and thus accelerate return to previous levels of function. The postoperative rehabilitation protocol lasts from 2 to 6 months and includes progressive steps toward full recovery (Table 1).

Traditional rehabilitation modalities are employed, including rest, ice, compression, and elevation, to reduce swelling and pain after surgery. Commencing range of motion exercises within the first week promotes ligament strength, decreases the chance of developing muscular atrophy or arthrofibrosis, and permits a quicker return to full activity. Initial range of motion exercises should be performed in the direction opposite that of the mechanism of injury to protect the ligament integrity. To further develop proprioception, the present authors advise early protected weight bearing in a boot. Before patients initiate strength exercises, $95 \%-100 \%$ of the pre-injury range of motion must be regained. From the present authors' experience, regaining full range of motion is most important within the first 6 weeks and will only change slightly past that point. External rotation is not permitted within the first 6 weeks in the setting of a deltoid tear. Dorsiflexion and eversion are also avoided until the ligament is healed.

Table I Postoperative rehabilitation protocol

\begin{tabular}{ll}
\hline Postoperative & Crutches; no weight bearing; elevate leg \\
& Walking boot and cold therapy \\
& Start home exercise program for stretching \\
I week & Home exercises (stretches and range-of-motion \\
& exercises) \\
& Protected weight bearing as tolerated \\
& Wean to one crutch \\
2 weeks & Assess range-of-motion \\
& Start home exercise with resistance bands \\
& Start weaning out of boot over next 2-4 weeks to \\
& stirrup brace, depending on comfort \\
I weeks & Normal gait in walking boot or brace \\
Increase in weight-bearing exercises
\end{tabular}

Note: Reproduced with permission from Porter DA: Evaluation and Treatment of Ankle Syndesmosis Injuries, in Azar FM, O'Connor MI (eds): Instructional Course Lectures, volume 58. Rosemont, IL, American Academy of Orthopaedic Surgeons, 2009. Abbreviation: BAPS, biomechanical ankle platform system. 
To diminish the likelihood of future or recurrent injuries to the ankle, proprioception regimens strengthen the muscles surrounding the ankle with functional exercises (ie, double leg stance, single leg stance, and kinesthetic awareness trainer). Activity or sport-specific functional progression allows constant evaluation of the patient and encouragement with signs of progress. This psychological reinforcement allows the patient to be an active member of the rehabilitation program. This series of progressively more difficult steps also reduces apprehension and builds confidence in the patient as they return to pre-injury activities.

The rehabilitation program can be divided into three phases. Phase I focuses on pain control, decreasing inflammation, and restoring normal joint range of motion (weeks 1-4). Phase II concentrates on foot and ankle flexibility and functional strengthening (weeks 4-8). Phase III emphasizes complete return to pre-injury functional activity (week 8 - full recovery). To increase ankle proprioception and confidence, the present authors require all athletes to wear an ankle brace for their first season of sport after syndesmosis fixation.

\section{Summary}

Although all patients experience a slightly different syndesmosis injury, every one desires an excellent functional outcome after surgery. The physician's duty is to restore preinjury stability, mobility, strength, and function as quickly and safely as possible. Any instability of the syndesmosis must be recognized and treated surgically with the key principles of anatomic reduction and secure fixation. Full rehabilitation typically requires 2-6 months and following an organized regimen that allows adequate time for healing with sequential, functional increases in activity.

\section{Disclosure}

David A Porter, MD, PhD, is a paid consultant to DJO Orthopedics. The authors have no other conflicts of interest in this work.

\section{References}

1. Clanton TO, Paul P. Syndesmosis injuries in athletes. Foot Ankle Clin N Am. 2002;7(3):529-549.

2. Lin CF, Gross MT, Weinhold P. Ankle syndesmosis injuries: anatomy, biomechanics, mechanism of injury, and clinical guidelines for diagnosis and intervention. J Orthop Sports Phys Ther. 2006;36(6): 372-384.

3. Mak MF, Gartner L, Pearce CJ. Management of syndesmosis injuries in the elite athlete. Foot Ankle Clin N Am. 2013;18(2):195-214.

4. Waterman BR, Belmont PJ Jr, Cameron KL, Svoboda SJ, Alitz CJ, Owens BD. Risk factors for syndesmotic and medial ankle sprain: role of sex, sport, and level of competition. Am J Sports Med. 2011;39(5): 992-998.
5. Press CM, Gupta A, Hutchinson MR. Management of ankle syndesmosis injuries in the athlete. Curr Sports Med Rep. 2009;8(5):228-233.

6. Porter DA. Evaluation and treatment of ankle syndesmosis injuries. Instructional Course Lectures. 2009;58:575-581.

7. Williams GN, Jones MH, Amendola A. Syndesmotic ankle sprains in athletes. Am J Sports Med. 2007;35(7):1197-1207.

8. Jelinek JA, Porter DA. Management of unstable ankle fractures and syndesmosis injuries in athletes. Foot Ankle Clin. 2009;14(2):277-298.

9. McCollum GA, van den Bekerom MP, Kerkhoffs GM, Calder JD, van Dijk CN. Syndesmosis and deltoid ligament injuries in the athlete. Knee Surg Sports Traumatol Arthrosc. 2013;21(6):1328-1337.

10. Mavi A, Yildirim H, Gunes H, Pestamalci T, Gumusburun E. The fibular incisura of the tibia with recurrent sprained ankle on magnetic resonance imaging. Saudi Med J. 2002;23(7):845-849.

11. Park JC, McLaurin TM. Acute syndesmosis injuries associated with ankle fractures: current perspectives in management. Bull NYU Hosp Jt Dis. 2009;67(1):39-44.

12. Ogilvie-Harris DJ, Reed SC, Hedman TP. Disruption of the ankle syndesmosis: biomechanical study of the ligamentous restraints. Arthroscopy. 1994;10(5):558-560.

13. Xenos JS, Hopkinson WJ, Mulligan ME, Olson EJ, Popovic NA. The tibiofibular syndesmosis: evaluation of the ligamentous structures, methods of fixation, and radiographic assessment. $J$ Bone Joint Surg Am. 1995;77(6):847-855.

14. Crim JR, Beals TC, Nickisch F, Schannen A, Saltzman CL. Deltoid ligament abnormalities in chronic lateral ankle instability. Foot Ankle Int. 2011;32(9):873-878.

15. Ramsey PL, Hamilton W. Changes in tibiotalar area of contact caused by lateral talar shift. J Bone Joint Surg. 1976;58(3):356-357.

16. van den Bekerom MP, Lamme B, Hogervorst M, Bolhuis HW. Which ankle fractures require syndesmotic stabilization? J Foot Ankle Surg. 2007;46(6):456-463.

17. Fites B, Kunes J, Madaleno J, Silvestri P, Johnson DL. Latent syndesmosis injuries in athletes. Orthopedics. 2006;29(2):124-127.

18. Leeds HC, Ehrlich MG. Instability of the distal tibiofibular syndesmosis after bimalleolar and trimalleolar ankle fractures. J Bone Joint Surg Am. 1984;66(4):490-503.

19. Nussbaum ED, Hosea TM, Sieler SD, Incremona BR, Kessler DE. Prospective evaluation of syndesmotic ankle sprains without diastasis. Am J Sports Med. 2001;29(1):31-35.

20. Weening B, Bhandari M. Predictors of functional outcome following transsyndesmotic screw fixation of ankle fractures. J Orthop Trauma. 2005;19(2):102-108.

21. Espinosa N, Smerek JP, Myerson MS. Acute and chronic syndesmosis injuries: pathomechanisms, diagnosis and management. Foot Ankle Clin. 2006;11(3):639-657.

22. Alonso A, Khoury L, Adams R. Clinical tests for ankle syndesmosis injury: reliability and prediction of return to function. J Orthop Sports Phys Ther. 1998;27(4):276-284.

23. Błasiak A, Sadlik B, Brzóska R. Injuries of the distal tibio-fibular syndesmosis. Pol Orthop Traumatol. 2013;78:139-150.

24. Taylor DC, Tenuta JJ, Uhorchak JM, Arciero RA. Aggressive surgical treatment and early return to sports in athletes with grade III syndesmosis sprains. Am J Sports Med. 2007;35(11):1833-1838.

25. Wikerøy AK, Høiness PR, Andreassen GS, Hellund JC, Madsen JE. No difference in functional and radiographic results 8.4 years after quadricortical compared with tricortical syndesmosis fixation in ankle fractures. J Orthop Trauma. 2010;24(1):17-23.

26. Han SH, Lee JW, Kim S, Suh JS, Choi YR. Chronic tibiofibular syndesmosis injury: the diagnostic efficiency of magnetic resonance imaging and comparative analysis of operative treatment. Foot Ankle Int. 2007;28(3):336-342.

27. Franke J, von Recum J, Suda AJ, Grützner PA, Wendl K. Intraoperative three-dimensional imaging in the treatment of acute unstable syndesmotic injuries. J Bone Joint Surg Am. 2012;94(15): 1386-1390.

28. Hintermann B, Knupp M, Pagenstert GI. Deltoid ligament injuries: diagnosis and management. Foot Ankle Clin. 2006;11(3):625-637. 
29. Phisitkul P, Ebinger T, Goetz J, Vaseenon T, Marsh JL. Forceps reduction of the syndesmosis in rotational ankle fractures: a cadaveric study. J Bone Joint Surg Am. 2012;94(24):2256-2261.

30. Tornetta P 3rd, Spoo JE, Reynolds FA, Lee C. Overtightening of the ankle syndesmosis: is it really possible? J Bone Joint Surg Am. 2001; 83-A(4):489-492.

31. Bragonzoni L, Russo A, Girolami M, et al. The distal tibiofibular syndesmosis during passive foot flexion. RSA-based study on intact, ligament injured and screw fixed cadaver specimens. Arch Orthop Trauma Surg. 2006;126(5):304-308.

32. Strömsöe K, Höqevold HE, Skjeldal S, Alho A. The repair of a ruptured deltoid ligament is not necessary in ankle fractures. J Bone Joint Surg Br. 1995;77(6):920-921.

33. Lambers KT, van den Bekerom MP, Doornberg JN, Stufkens SA, van Dijk CN, Kloen P. Long-term outcome of pronation-external rotation ankle fractures treated with syndesmotic screws only. J Bone Joint Surg Am. 2013;95(17):e1221-e1227.

34. Schepers T. Acute distal tibiofibular syndesmosis injury: a systematic review of suture-button versus syndesmotic screw repair. Int Orthop. 2012;36(6):1199-1206.
35. Schepers T. To retain or remove the syndesmotic screw: a review of literature. Arch Orthop Trauma Surg. 2011;131(7):879-883.

36. Coetzee JC, Ebeling P. Treatment of syndesmosis disruptions with tightrope fixation. Tech Foot Ankle Surg. 2008;7(3):196-202.

37. Qamar F, Kadakia A, Venkateswaran B. An anatomical way of treating ankle syndesmotic injuries. J Foot Ankle Surg. 2011;50(6):762-765.

38. Naqvi GA, Shafqat A, Awan N. Tightrope fixation of ankle syndesmosis injuries: clinical outcome, complications and technique modification. Injury. 2012;43(6):838-842.

39. Willmott HJ, Singh B, David LA. Outcome and complications of treatment of ankle diastasis with tightrope fixation. Injury. 2009;40(11):1204-1206.

40. Cottom JM, Hyer CF, Philbin TM, Berlet GC. Treatment of syndesmotic disruptions with the Arthrex Tightrope: a report of 25 cases. Foot Ankle Int. 2008;29(8):773-780.

41. Thornes B, Shannon F, Guiney AM, Hession P, Masterson E. Suture-button syndesmosis fixation: accelerated rehabilitation and improved outcomes. Clin Orthop Relat Res. 2005;(431):207-212.
Open Access Journal of Sports Medicine

\section{Publish your work in this journal}

Open Access Journal of Sports Medicine is an international, peer-reviewed, open access journal publishing original research, reports, reviews and commentaries on all areas of sports medicine. The manuscript management system is completely online and includes a very quick and fair peer-review system.

\section{Dovepress}

Visit http://www.dovepress.com/testimonials.php to read real quotes from published authors. 